\title{
Global Multiobjective Optimization via Estimation of Distribution Algorithm with Biased Initialization and Crossover
}

\author{
Aiming Zhou \\ Department of Computer \\ Science \\ University of Essex \\ Colchester, CO4 3SQ, U. K. \\ azhou@essex.ac.uk
}

\author{
Qingfu Zhang \\ Department of Computer \\ Science \\ University of Essex \\ Colchester, CO4 3SQ, U. K. \\ qzhang@essex.ac.uk
}

\author{
Yaochu Jin \\ Honda Research Institute \\ Europe \\ Carl-Legien-Str.30,63073 \\ Offenbach,Germany \\ yaochu.jin@honda-ri.de
}

Bernhard Sendhoff
Honda Research Institute
Europe
Carl-Legien-Str.30,63073
Offenbach,Germany
bernhard.sendhoff@honda-

ri.de

\author{
Edward Tsang \\ Department of Computer \\ Science \\ University of Essex \\ Colchester, CO4 3SQ, U. K. \\ edward@essex.ac.uk
}

\begin{abstract}
Multiobjective optimization problems with many local Pareto fronts is a big challenge to evolutionary algorithms. In this paper, two operators, biased initialization and biased crossover, are proposed to improve the global search ability of RM-MEDA, a recently proposed multiobjective estimation of distribution algorithm. Biased initialization inserts several globally Pareto optimal solutions into the initial population; biased crossover combines the location information of some best solutions and globally statistical information in the current population. Experiments have been conducted to study the effects of these two operators.
\end{abstract}

\section{Categories and Subject Descriptors}

I.2.8 [Artificial intelligence]: Problem Solving, Control Methods, and Search

\section{General Terms}

Algorithm

\section{Keywords}

estimation of distribution algorithm, global optimization, multiobjective optimization, biased initialization, biased crossover

\section{INTRODUCTION}

Permission to make digital or hard copies of all or part of this work for personal or classroom use is granted without fee provided that copies are not made or distributed for profit or commercial advantage and that copies bear this notice and the full citation on the first page. To copy otherwise, to republish, to post on servers or to redistribute to lists, requires prior specific permission and/or a fee.

Copyright 200X ACM X-XXXXX-XX-X/XX/XX ...\$5.00.
Global optimization by evolutionary algorithms (EAs) has been widely studied for scalar objective optimization problems $[9,12,13,16]$. However, there is not much effort on it for multiobjective optimization problems (MOPs) $[4,8]$. Strategies for global scalar objective optimization may not be steadily extended to multiobjective optimization evolutionary algorithms (MOEAs).

Estimation of distribution algorithms (EDAs) are a new computing paradigm in evolutionary computation [7]. There is no crossover or mutation in EDAs. Instead, they explicitly extract globally statistical information from the selected solutions and build a posterior probability distribution model of promising solutions, based on the extracted information. New solutions are sampled from the model thus built and fully or in part replace the old population. The Pareto set (PS) of a continuous MOP is a piecewise continuous $(m-1)$ $\mathrm{D}$ manifold. This property has been used in several mathematical programming methods. However, such regularity has not yet been exploited by evolutionary algorithms. Recently, we proposed RM-MEDA [17, 18, 19, 20], a regularity model based EDA for continuous MOPs. Experimental results have shown that RM-MEDA can effectively deal with variable linkages. However, if a MOP has many local Pareto fronts (PFs), RM-MEDA could fail in locating the global $\mathrm{PF}$.

This paper introduces two new operators, i.e., biased initialization and biased crossover, for improving the global search ability of RM-MEDA. These two operators aim at guiding the search toward the global PS. Biased initialization inserts several globally Pareto optimal solutions into the initial population; biased crossover combines the location information of some best solutions and globally statistical information in the current population.

The rest of the paper is organized as follows. Section 2 gives some notations and definitions. In Section 3, RMMEDA is briefly described. The biased initialization and biased crossover are introduced in Section 4. In Section 5, the experimental results are presented. And the paper is 
concluded in Section 6.

\section{NOTATIONS AND DEFINITIONS}

We consider the following continuous MOP:

$$
\min F(X)=\left(f_{1}(X), \cdots, f_{m}(X)\right)^{T}
$$

where $X \in \Omega \subseteq R^{n}, \Omega$ is a continuous search space, and each objective $\bar{f}_{i}: \Omega \rightarrow R$ is continuous of decision variable $X$. Very often, the objectives in a MOP conflict with each other, no single solution can optimize all the objectives at the same time. Pareto optimality is used for defining the best trade-off solutions of a MOP.

A vector $u \in R^{m}$ dominates another vector $v \in R^{m}$, denoted as $u \prec v$, iff $u_{i} \leq v_{i}$ for all $i=1, \cdots, m$ and $u_{j}<v_{j}$ for at least one $j \in\{1, \cdots, m\}$. The following two definitions are based on this Pareto domination.

Definition 1 (Local Pareto Set): For a given $\varepsilon>0$, a local Pareto set of (1) is a set of solutions $X \in \Omega$ which can not be dominated by other solution $Y \in \Omega$ which satisfies $\|X-Y\|<\varepsilon$. Mathematically, it can be denoted as $L P S=$ $\{X \mid X \in \Omega, \nexists Y \in \Omega,\|X-Y\|<\varepsilon, F(Y) \prec F(X)\}$.

Definition 2 (Global Pareto Set): Global Pareto set of (1) contains all optimal solutions which can not be dominated by any solutions in the search space and it can be denoted as $G P S=\{X \mid X \in \Omega, \nexists Y \in \Omega, F(Y) \prec F(X)\}$.

The image of local Pareto set and global Pareto set in objective space are called local Pareto front and global Pareto front, denoted as LPF and GPF respectively.

MOEAs for global optimization aim to find an approximation of the GPS and GPF of (1).

\section{THE FRAMEWORK OF RM-MEDA}

Under some mild conditions, the PS of (1) defines a $(m-$ 1 )-dimensional manifold where $m$ is the number of objectives.

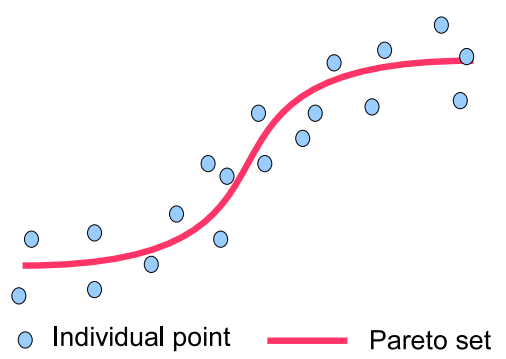

Figure 1: Illustration of individual solutions scattered around the PS in the decision space.

As shown in Figure 1, the population in the decision space in an ideal MOEA for (1) will hopefully approximate the PS and be uniformly scattered around the PS as the search goes on. Therefore, we can envisage the points in the population as independent observations of a random vector $\xi \in R^{n}$ whose centroid is the PS of (1) and can be naturally described by:

$$
\xi=\zeta+\varepsilon
$$

where $\zeta$ is uniformly distributed over a piecewise continuous $(m-1)$-dimensional manifold. $\varepsilon$ is an $n$-dimensional zeromean noise vector and $n$ is the number of decision variables.
In RM-MEDA, piecewise $(m-1)$-dimensional linear models are used to approximate model $\zeta$ in (2). Local principal component analysis [5] is applied to partition a population. In each cluster, the parameters of linear model and noise, $\varepsilon$ in (2), are estimated by principal component analysis. New trial solutions are than sampled from model (2).

Let $P_{t}$ denote a population at generation $t, P_{t}^{O}$ denote the offspring generated at generation $t$ and $P_{t}^{N}$ denote all the nondominated solutions in $P_{t}$. The size of both $P_{t}$ and $P_{t}^{O}$ is fixed to be $N$. The algorithm works as follows:

\section{RM-MEDA}

Step 0: Set $t:=0$. Generate an initial population $P_{0}$ and evaluate $P_{0}$.

Step 1: If stopping condition is met, stop and return $P_{t}^{N}$ which constitutes an approximation to the PF (PS).

Step 2: Build the probability model (2) for modelling the distribution of the solutions in $P_{t}$.

Step 3: Sample a new solution set $P_{t}^{O}$ from the model (2) and evaluate $P_{t}^{O}$.

Step 4: Select $N$ individuals from $P_{t}^{O} \cup P_{t}$ to create $P_{t+1}$.

Step 5: Set $t:=t+1$ and go to Step 1 .

In the framework of RM-MEDA, the population is initialized randomly in the search space in Step $\mathbf{0}$ and a modified version of selection based on the nondominated sorting and crowding distance of NSGA-II [1] is used in Step 4. The algorithm will stop according to a predefined maximal function evaluations. More details of RM-MEDA can be found in $[17]$.

\section{BIASED OPERATORS}

\subsection{Biased Initialization}

It is not a new idea to add some 'good' points in initial population to improve the performance of MOEAs. This strategy makes MOEAs like two stage search methods: in stage I, efforts are spent on finding solutions near/on PF while in stage II, the whole PF is generated. In $[6,10]$, by optimizing a few of aggregation functions with deterministic gradient based optimization methods, a few points, called supporting solutions, are put into an initial population to improve the performance of MOEAs. In [11], a two-phase local search is designed for bi-objective traveling salesman problems. In stage I, an initial solution is generated by optimizing only one single objective, and then in stage II, the whole $\mathrm{PF}$ is generated by optimizing a sequence of scalar objective problems based on aggregations of the objectives. In [3], the algorithm focuses on finding one solution near PF by a method similar to $(1+1)$-ES in stage I and in stage II, a steady state EA is used to spread the individuals along the PF.

For global multiobjective optimization, 'good' points near/on $\mathrm{PF}$ might (a) prevent population from trapping onto local PF because these 'good' points will dominate some points on local PF, and (b) guide population to global population if they are used in generating offspring.

In this paper, only $m$ ( $m$ is the number of objective) points are generated by an EA for global optimization. 
There are many ways to convert a MOP into a single objective optimization problem [14]. In this paper, the first $m$ initial solutions are generated as follows:

$$
X_{i}=\operatorname{argmin}_{X \in \Omega} \sum_{j=1}^{m} \alpha_{j}^{i} f_{j}(X)
$$

where $i=1, \cdots, m, \alpha_{j}^{i}$ are randomly chosen weights which satisfy $0<\alpha_{j}^{i}<1$ and $\sum_{j=1}^{m} \alpha_{j}^{i}=1$.

All the other initial solutions are randomly sampled from the decision space:

$$
X_{i}=\operatorname{rand}(\Omega)
$$

where $i=m+1, \cdots, N$ and $N$ is the population size. $\operatorname{rand}(\Omega)$ returns a uniformly random point in $\Omega$.

In this initialization, $m$ initial solutions will hopefully be near/on the global PF (PS).

\subsection{Biased Crossover}

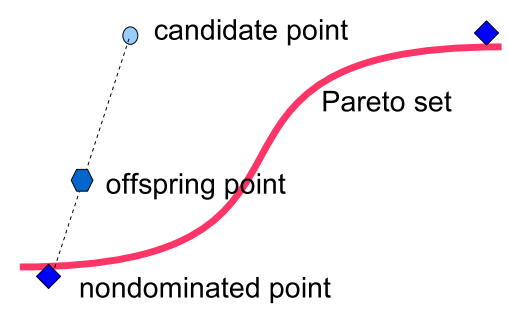

Figure 2: Illustration of biased crossover.

One of the major shortcoming of an EDA is that modelling may ignore isolated high-quality individuals. To overcome this shortcoming, guided mutation has been proposed by combining location information of individuals and global statical information [15]. Biased crossover uses the same idea and tries to keep the influence of the best solutions particularly when they are few.

Let $P_{t}^{E}$ be a set of solutions generated from $P_{t}$ by the EDA operator. The biased crossover generates a set of new solutions, $P_{t}^{O}$, in the following way:

\section{Biased Crossover}

Step 1: Select the non-dominated set $P_{t}^{N}$ from $P_{t}$, and set $P_{t}^{O}$ empty.

Step 2: If $\frac{\left|P_{t}^{N}\right|}{\left|P_{t}\right|}>\theta$, set $P_{t}^{O}=P_{t}^{E}$ and stop, else go to Step 3.

Step 3: For each point $X^{E} \in P_{t}^{E}$, randomly select a point $X^{N} \in P_{t}^{N}$, generate a new point

$$
X=X^{E}+\beta\left(X^{N}-X^{E}\right)
$$

where $\beta \in[0,1]$ is a random number, and put it into offspring set $P_{t}^{O}=P_{t}^{O} \cup\{X\}$.

In our experiments, the threshold is fixed to be 0.2. In the above operator, when the size of $P_{t}^{N}$ is small compared with that of $P_{t}$ (i.e., $\frac{\left|P_{t}^{N}\right|}{\left|P_{t}\right|} \leq \theta$ ), all the new solutions are recombined with solutions in $P_{t}^{N}$. On the other hand, if nondominated solutions have a large fraction in the population, the population distribution model will represent these individuals and thus there is no need to emphasize them again in biased crossover.

In Step 3, a new solution is generated between a reference nondominated point and a candidate point which is illustrated in Figure 2.

\subsection{Enhanced RM-MEDA for Global Optimiza- tion}

The above two biased operators can be incorporated into RM-MEDA for global optimization and the resultant method works as follows:

\section{Enhanced RM-MEDA for Global Optimization}

Step 0: Set $t:=0$. Generate an initial population $P_{0}$ by biased initialization.

Step 1: If stopping condition is met, stop and return $P_{t}^{N}$ which constitutes an approximation to the PF (PS).

Step 2: Build the probability model (2) for modelling the distribution of the solutions in $t$.

Step 3: Generate a candidate solution set $P_{t}^{E}$ from the model (2).

Step 4: Generate an offspring set $P_{t}^{O}$ from $P_{t}$ and $P_{t}^{E}$ via biased crossover and and evaluate $P_{t}^{O}$.

Step 5: Select $N$ individuals from $P_{t}^{O} \cup P_{t}$ to create $P_{t+1}$.

Step 6: Set $t:=t+1$ and go to Step 1 .

The only differences between the original of RM-MEDA and the above method in Section 3 are in Step 0 and Step 4 where biased initialization and biased crossover are used respectively.

\section{EXPERIMENTAL RESULTS}

We have conducted experimental studies on several test instances. In the following, we report our results on the following modified ZDT4 [1] and DTLZ3 [2]:

$$
Z D T 4\left\{\begin{array}{l}
f_{1}(X)=x_{1} \\
f_{2}(x)=g(x)\left[1-\sqrt{f_{1}(x) / g(x)}\right]
\end{array}\right.
$$

where $X \in[0,1] \times[0,10]^{9}$ and $g$ used in the experiments are

$$
g_{1}(x)=\frac{1}{4000} \sum_{i=2}^{10}\left(x_{i}^{2}-x_{1}\right)^{2}-\prod_{i=2}^{10} \cos \left(\frac{x_{i}^{2}-x_{1}}{\sqrt{i-1}}\right)+2
$$

and

$$
g_{2}(x)=91+\sum_{i=2}^{10}\left[\left(x_{i}^{2}-x_{1}\right)^{2}-10 \cos \left(2 \pi\left(x_{i}^{2}-x_{1}\right)\right)\right] .
$$

The instances of ZDT4 with $g_{1}$ and $g_{2}$ are denoted as $F_{1}$ and $F_{2}$, respectively.

$$
D T L Z 3\left\{\begin{array}{l}
f_{1}(X)=(1+g(X)) \cos \left(x_{1} \pi / 2\right) \cos \left(x_{2} \pi / 2\right) \\
f_{2}(X)=(1+g(X)) \cos \left(x_{1} \pi / 2\right) \sin \left(x_{2} \pi / 2\right) \\
f_{3}(X)=(1+g(X)) \sin \left(x_{1} \pi / 2\right)
\end{array}\right.
$$


Table 1: Mean and Std. of $D$ and $\Upsilon$ achieved on $F_{1}$ and $F_{2}$.

\begin{tabular}{|c|c|c|c|c|}
\hline & \multicolumn{2}{|c|}{$F_{1}$} & \multicolumn{2}{c|}{$F_{2}$} \\
\hline & $D$ & $\Upsilon$ & $D$ & $\Upsilon$ \\
\hline A1 & $0.0193(0.0114)$ & $0.0182(0.0119)$ & $3.0120(2.7929)$ & $2.7581(2.9146)$ \\
\hline A2 & $0.0383(0.0195)$ & $0.0372(0.0196)$ & $132.3238(126.8185)$ & $133.2056(127.8889)$ \\
\hline A3 & $0.0261(0.0177)$ & $0.0246(0.0174)$ & $159.7894(177.7885)$ & $161.5801(180.1942)$ \\
\hline A4 & $\mathbf{0 . 0 0 4 3}(0.0001)$ & $\mathbf{0 . 0 0 2 0}(0.0001)$ & $\mathbf{0 . 1 1 1 8}(0.2376)$ & $\mathbf{0 . 0 4 3 1}(0.1902)$ \\
\hline
\end{tabular}

where $X \in[0,1]^{2} \times[0,10]^{8}$ and $g$ used in the experiments are

$$
g_{3}(x)=\frac{1}{4000} \sum_{i=3}^{10}\left(x_{i}^{2}-x_{1}\right)^{2}-\prod_{i=3}^{10} \cos \left(\frac{x_{i}^{2}-x_{1}}{\sqrt{i-2}}\right)+1
$$

and

$$
g_{4}(x)=81+\sum_{i=3}^{10}\left[\left(x_{i}^{2}-x_{1}\right)^{2}-10 \cos \left(2 \pi\left(x_{i}^{2}-x_{1}\right)\right)\right] .
$$

The instances of DTLZ3 with $g_{3}$ and $g_{4}$ are denoted as $F_{3}$ and $F_{4}$, respectively.

D-metric [17] and $\Upsilon$-metric [1] are used here to measure the performance. Let $P^{*}$ be a set of uniformly distributed points in the objective space along the $\mathrm{PF}$, and let $P$ be an approximation to the PF. D-metric and $\Upsilon$-metric are defined as:

$$
\begin{aligned}
& D\left(P^{*}, P\right)=\frac{\sum_{v \in P^{*}} d(v, P)}{\left|P^{*}\right|} \\
& \Upsilon\left(P, P^{*}\right)=\frac{\sum_{v \in P} d\left(v, P^{*}\right)}{|P|}
\end{aligned}
$$

where $d(a, A)$ is the minimum Euclidean distance between $a$ and the points in $A$. If $\left|P^{*}\right|$ is large enough to represent the PF very well, $D\left(P^{*}, P\right)$ could measure both the diversity and convergence of $P$ in a sense when $P$ is close to $P^{*}$, while $\Upsilon\left(P, P^{*}\right)$ only measures the convergence of $P$.

In our experiments, we select 500 evenly distributed points on $\mathrm{PF}$ and let these points be $P^{*}$ for each test instance with 2 objectives, and 1,000 points for each test instance with 3 objectives.

In the following, RM-MEDA, RM-MEDA with biased initialization, RM-MEDA with biased crossover, and RM-MEDA with both biased operators are denoted as $A 1, A 2, A 3$, and $A 4$, respectively.

The parameters are as follows: for bi-objective problems $F_{1}$ and $F_{2}$, the population size is 100 ; maximal function evaluation is 40,000 for (among which 20,000 is used by EDA/L [16] in biased initialization if necessary); the weights used in biased initialization are fixed to $\alpha=(0.9,0.1)$ and $\alpha=(0.1,0.9)$. For tri-objective problems $F_{3}$ and $F_{4}$, the population size is 200; maximal function evaluation is 70,000 for (among which 30,000 is used by EDA/L in biased initialization if necessary); the weights used in biased initialization are fixed to $\alpha=(0.8,0.1,0.1), \alpha=(0.1,0.8,0.1)$ and $\alpha=(0.1,0.1,0.8)$. In all executions, the cluster number in RM-MEDA is 5 . The results are based on 100 independent runs.

\subsection{Results for modified ZDT4 problems}

The mean and standard deviation of the two metrics are shown in Table 1. The PFs obtained by the four algorithms in the final generation are shown in Figure 3.
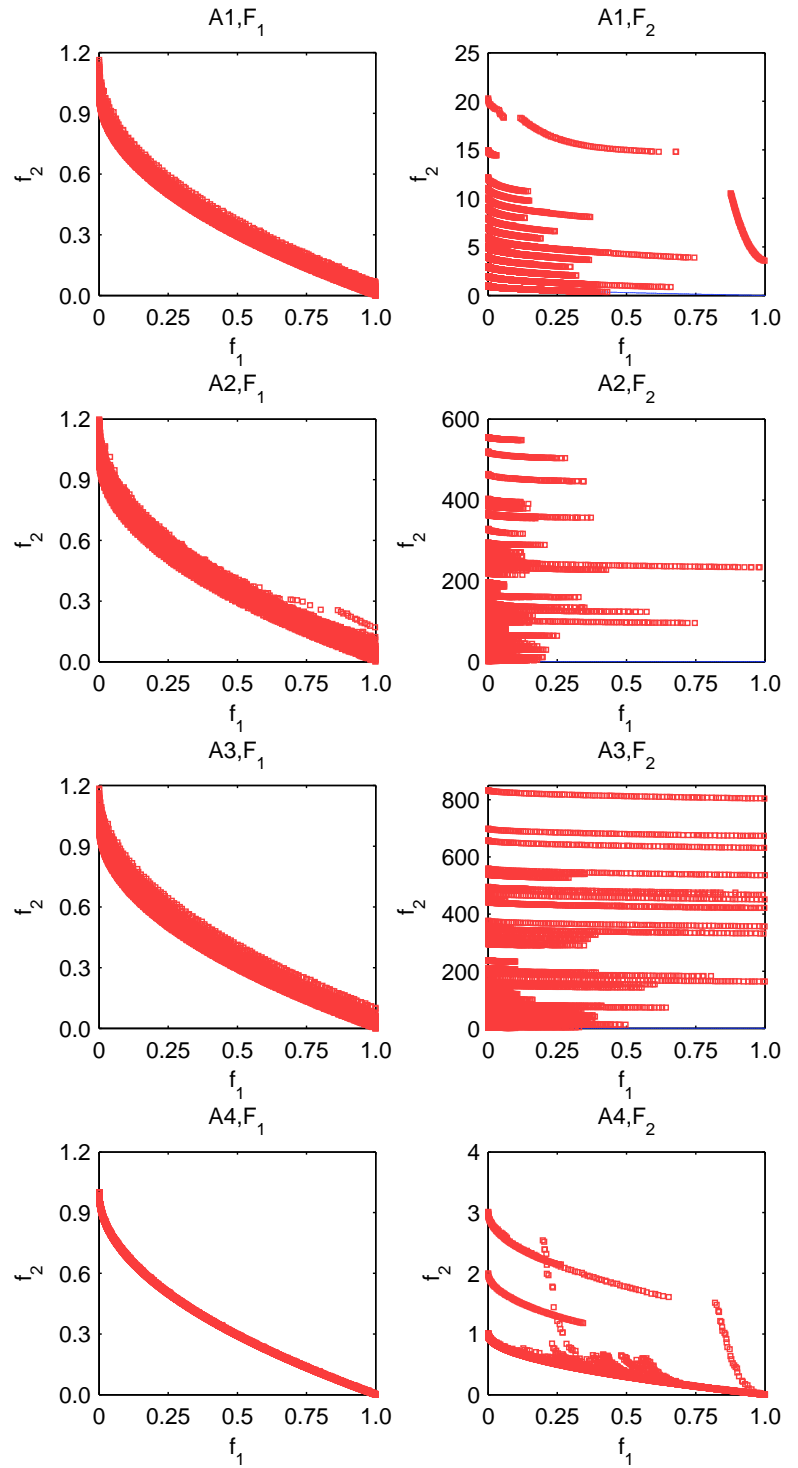

Figure 3: Pareto fronts obtained by the four algorithms on $F_{1}$ and $F_{2}$. 
Table 2: Mean and Std. of $D$ and $\Upsilon$ achieved on $F_{3}$ and $F_{4}$.

\begin{tabular}{|c|c|c|c|c|}
\hline & \multicolumn{2}{|c|}{$F_{3}$} & \multicolumn{2}{c|}{$F_{4}$} \\
\hline & $D$ & $\Upsilon$ & $D$ & $\Upsilon$ \\
\hline A1 & $0.0617(0.0044)$ & $0.0514(0.0118)$ & $2.9890(4.8322)$ & $10454.9999(17126.2022)$ \\
\hline A2 & $0.0629(0.0053)$ & $0.0531(0.0120)$ & $0.5949(0.1566)$ & $6505.2868(14582.7179)$ \\
\hline A3 & $\mathbf{0 . 0 6 0 8}(0.0042)$ & $\mathbf{0 . 0 4 8 9}(0.0113)$ & $0.9853(2.0288)$ & $2813.9000(9869.7266)$ \\
\hline A4 & $0.0612(0.0046)$ & $0.0508(0.0132)$ & $\mathbf{0 . 4 9 9 0}(0.0400)$ & $\mathbf{0 . 0 4 1 0}(0.0955)$ \\
\hline
\end{tabular}

The results in Table 1 show that if only the biased initialization or biased crossover is used, the performance of RM-MEDA will become poorer on both test instances. The reason is that for $A 2$, although some good solutions are put into the initial population, they don't play any role in the EDA operator; and for $A 3$, the biased crossover might mislead the population into local PFs and it is why A3 failed in $F_{2}$, as shown in Figure 3. By using both biased initialization and biased crossover, the performance of RM-MEDA has been significantly improved. It can also be seen from Figure. 3, that the final PFs of $A 4$ are closer to the global PFs than those of $A 1$.

\subsection{Results for modified DTLZ3 problems}

The mean and standard deviation of the two metrics are shown in Table 2. The PFs obtained by the four algorithms in the final generation are shown in Figure 4.

The results show that A3 performs slightly better than A4 on F3.

For $F_{4}$, it is clear that only A4 can converge to the global $\mathrm{PF}$ in most of runs. The $\Upsilon$-metric values indicate that Pareto fronts achieved by the other three algorithms are still far away from global Pareto front.

\section{CONCLUSIONS}

In this paper, a biased initialization and a biased crossover have been introduced to improve the global search ability of RM-MEDA. In biased initialization, by solving several scalar objective optimization problems converted from a multiobjective optimization problem, some 'good' points are generated near/on global Pareto front. These 'good' points will then prevent the population from trapping into local Pareto fronts and guide the population into global Pareto front by biased crossover.

The proposed strategy is tested on bi-objective and triobjective problems and the results show that the global search ability of RM-MEDA is improved remarkably compared to RM-MEDA and RM-MEDA with only one of the biased operators.

To achieve an optimal approximation of global Pareto front, the costs used in initialization and in the main evolving process should be balanced. In this paper, the costs used in two stages are fixed. A more practical way should allocate the cost in these two phases adaptively. And this will be our future work.

\section{REFERENCES}

[1] K. Deb, A. Pratap, S. Agarwal, and T. Meyarivan. A fast and elitist multiobjective genetic algorithm: NSGA-II. IEEE Transactions on Evolutionary Computation, 6(2):182-197, 2002.

[2] K. Deb, L. Thiele, M. Laumanns, and E. Zitzler. Scalable Test Problems for Evolutionary
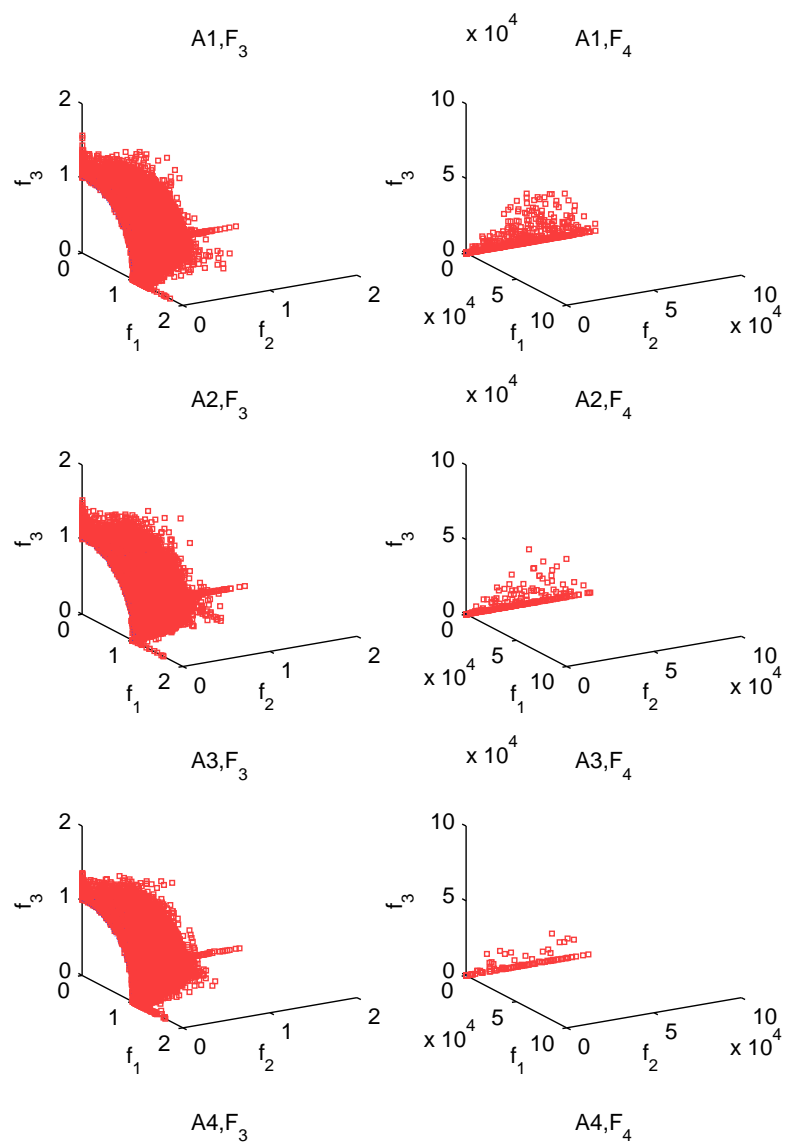

$\times 10^{4} \quad \mathrm{~A}_{3}, \mathrm{~F}_{4}$
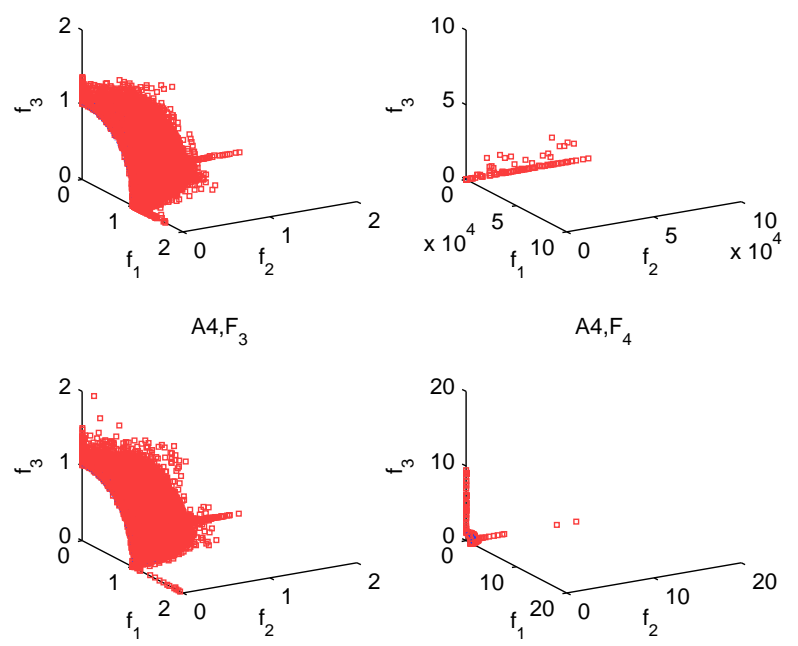

Figure 4: Pareto fronts obtained by the four algorithms on $F_{3}$ and $F_{4}$. 
Multiobjective Optimization. In A. Abraham, L. Jain, and R. Goldberg, editors, Evolutionary Multiobjective Optimization. Theoretical Advances and Applications, pages 105-145. Springer, USA, 2005.

[3] C. Groşan. Multiobjective adaptive representation evolutionary algorithm (marea) - a new evolutionary algorithm for multiobjective optimization. In Applied Soft Computing Technologies: The Challenge of Complexity, Advances in Soft Computing, volume 34 of Advances in Soft Computing, pages 113-121. Springer-Verlag, 2006.

[4] T. Hanne. Global multiobjective optimization using evolutionary algorithms. Journal of Heuristics, 6(3):347-360, 2000.

[5] N. Kambhatla and T. K. Leen. Dimension reduction by local principal component analysis. Neural Computation, 9(7):1493-1516, October 1997.

[6] M. Lahanas, E. Schreibmann, N. Milickovic, and D. Baltas. Intensity modulated beam radiation therapy dose optimization with multiobjective evolutionary algorithms. In Second International Conference on Evolutionary Multi-Criterion Optimization (EMO 2003), volume 2632 of Lecture Notes in Computer Science, pages 648-661, Faro, Portugal, April 2003. Springer.

[7] P. Larrañaga and J. A. Lozano, editors. Estimation of Distribution Algorithms : A New Tool for Evolutionary Computation. Kluwer Academic Publishers, Norwell, MA, USA, 2001.

[8] M. Laumanns and J. Očenášek. Bayesian optimization algorithms for multi-objective optimization. In Parallel Problem Solving From Nature - PPSN VII, volume 2439 of Lecture Notes in Computer Science, pages 298-307, Granada,Spain, September 2002. Springer.

[9] Y. W. Leung and Y. Wang. An orthogonal genetic algorithm with quantization for global numerical optimization. IEEE Transactions on Evolutionary Computation, 5(1):41-53, 2001.

[10] N. Milickovic, M. Lahanas, D. Baltas, and N. Zamboglou. Comparison of evolutionary and deterministic multiobjective algorithms for dose optimization in brachytherapy. In E. Zitzler, K. Deb, L. Thiele, C. A. Coello Coello, and D. Corne, editors, First International Conference on Evolutionary Multi-Criterion Optimization (EMO 2001), volume 1993 of Lecture Notes in Computer Science, pages 167-180, Zurich, Switzerland, March 2001. Springer.

[11] L. Paquete and T. Stüzle. A two-phase local search for the biobjective traveling salesman problem. In Second International Conference on Evolutionary Multi-Criterion Optimization (EMO 2003), volume 2632 of Lecture Notes in Computer Science, pages 479-493, Faro, Portugal, April 2003. Springer.

[12] J. T. Tsai, T. K. Liu, and J. H. Chou. Hybrid taguchi-genetic algorithm for global numerical optimization. IEEE Transactions on Evolutionary Computation, 8(4):365-377, 2004.

[13] Z. Tu and Y. Lu. A robust stochastic genetic algorithm (StGA) for global numerical optimization. IEEE Transactions on Evolutionary Computation, 8(5):456-470, 2004.

[14] Q. Zhang and H. Li. A multi-objective evolutionary algorithm based on decomposition. IEEE Transactions on Evolutionary Computation, 2007. accepted for publication.

[15] Q. Zhang, J. Sun, and E. Tsang. Evolutionary algorithm with the guided mutation for the maximum clique problem. IEEE Transactions on Evolutionary Computation, 9(2):1-9, April 2005.

[16] Q. Zhang, J. Sun, E. Tsang, and J. Ford. Hybrid stimation of distribution algorithm for global optimisation. Engineering Computations, 21(1):91-107, 2003.

[17] Q. Zhang, A. Zhou, and Y. Jin. Modelling the regularity in estimation of distribution algorithm for continuous multi-objective evolutionary optimization with variable linkages. IEEE Transactions on Evolutionary Computation, 2007. accepted for publication.

[18] A. Zhou, Y. Jin, Q. Zhang, B. Sendhoff, and E. Tsang. Combining model-based and genetics-based offspring generation for multi-objective optimization using a convergence criterion. In Proceedings of the Congress on Evolutionary Computation (CEC 2006), pages 3234-3241, Vancouver, BC, Canada, July 2006. IEEE Press.

[19] A. Zhou, Q. Zhang, Y. Jin, B. Sendhoff, and E. Tsang. Modelling the population distribution in multi-objective optimization by generative topographic mapping. In Parallel Problem Solving From Nature (PPSN IX), volume 4193 of Lecture Notes in Computer Science, pages 443-452, Reykjavik, Iceland, September 2006. Springer-Verlag.

[20] A. Zhou, Q. Zhang, Y. Jin, E. Tsang, and T. Okabe. A model-based evolutionary algorithm for bi-objective optimization. In Proceedings of the Congress on Evolutionary Computation (CEC 2005), pages 2568-2575, Edinburgh, U.K, September 2005. IEEE Press. 